Ferrata Storti Foundation

\title{
Bortezomib-based induction followed by stem cell transplantation in light chain amyloidosis: results of the multicenter HOVON 104 trial
}

Haematologica 2019

Volume 104(11):2274-2282

\section{Correspondence: \\ MONIQUE C. MINNEMA \\ m.c.minnema@umcutrecht.nl \\ Received: December 6, 2018. \\ Accepted: March 18, 2019. \\ Pre-published: March 28, 2019.}

doi:10.3324/haematol.2018.213900

Check the online version for the most updated information on this article, online supplements, and information on authorship \& disclosures: www.haematologica.org/content/104/11/2274

\section{(C)2019 Ferrata Storti Foundation}

Material published in Haematologica is covered by copyright. All rights are reserved to the Ferrata Storti Foundation. Use of published material is allowed under the following terms and conditions:

https://creativecommons.org/licenses/by-nc/4.0/legalcode. Copies of published material are allowed for personal or internal use. Sharing published material for non-commercial purposes is subject to the following conditions:

https://creativecommons.org/licenses/by-nc/4.0/legalcode, sect. 3. Reproducing and sharing published material for commercial purposes is not allowed without permission in writing from the publisher.

\author{
Monique C. Minnema, ${ }^{1}$ Kazem Nasserinejad, ${ }^{2}$ Bouke Hazenberg, ${ }^{3}$ Ute \\ Hegenbart, ${ }^{4}$ Philip Vlummens, ${ }^{5}$ Paula F. Ypma, ${ }^{6}$ Nicolaus Kröger, ${ }^{7}$ Ka Lung \\ Wu, ${ }^{8}$ Marie Jose Kersten, ${ }^{9}$ M. Ron Schaafsma, ${ }^{10}$ Sandra Croockewit, ${ }^{11}$ Esther \\ de Waal, ${ }^{12}$ Sonja Zweegman, ${ }^{13}$ Lidwien Tick, ${ }^{14}$ Annemieke Broijl, ${ }^{15}$ Harry \\ Koene, ${ }^{16}$ Gerard Bos, ${ }^{17}$ Pieter Sonneveld ${ }^{15}$ and Stefan Schönland ${ }^{4}$
} ${ }^{1}$ Department of Hematology, UMC Utrecht Cancer Center, Utrecht, the Netherlands;
${ }^{2}$ HOVON Data Center, Department of Hematology, Erasmus MC Cancer Institute, Rotterdam, the Netherlands; ${ }^{3}$ Department of Rheumatology \& Clinical Immunology, University of Groningen Medical Center, Groningen, the Netherlands; ${ }^{4}$ Department of Hematology, Oncology and Rheumatology, Heidelberg University, Amyloidosis Center, Heidelberg, Germany; ${ }^{5}$ Department of Haematology, Ghent University, Gent, Belgium; ${ }^{6}$ Department of Hematology, HagaZiekenhuis, Den Haag, the Netherlands; ' Department of Stem Cell Transplantation, University Medical Center Hamburg-Eppendorf, Hamburg, Germany; ${ }^{8}$ Department of Hematology, ZNA Stuivenberg, Antwerp, Belgium; ${ }^{9}$ Department of Hematology, Academic Medical Center, Lymphoma and Myeloma Center, Amsterdam, the Netherlands; ${ }^{10}$ Department of Hematology, Medisch Spectrum Twente, Enschede, the Netherlands; ${ }^{11}$ Department of Hematology, Radboud University Medical Center, Nijmegen, the Netherlands; ${ }^{12}$ Department of Hematology, University of Groningen, Medical Center, Groningen, the Netherlands; ${ }^{13}$ Department of Hematology, VU University Medical Center, Amsterdam Cancer Center, Amsterdam, the Netherlands; ${ }^{14}$ Department of Internal Medicine, Maxima Medisch Centrum, Eindhoven, the Netherlands; ${ }^{15}$ Department of Hematology, Erasmus MC Cancer Institute, Rotterdam, the Netherlands; ${ }^{16}$ Department of Hematology, St. Antonius Hospital, Nieuwegein, the Netherlands and ${ }^{17}$ Department of Hematology, Maastricht University Medical Center, Maastricht, the Netherlands 


\section{Introduction}

Light chain (AL) amyloidosis is a potentially fatal disorder caused by a small monoclonal population of plasma cells in the bone marrow, which synthesize monoclonal light chains. The light chains are considered toxic and aggregate into amyloid fibrils. These fibrils form extracellular deposits in one or more vital organs, most frequently in the kidneys, heart and liver as well as in the peripheral and autonomic nervous systems. ${ }^{1}$

The main goal of treatment is to reduce the supply of the amyloidogenic monoclonal light chains rapidly and to achieve a very good partial response (VGPR) or a complete hematologic response (CR). ${ }^{2}$ Achieving a hematologic response is closely related with survival. This has been demonstrated following both high-dose and standard-dose chemotherapy.,3 Organ responses are also induced in patients with prolonged hematologic responses.

High-dose melphalan (HDM) followed by autologous SCT (auto-SCT) is considered the most effective treatment for selected patients with AL amyloidosis. ${ }^{4}$ However, this treatment is only feasible in around $20 \%$ or fewer of newly diagnosed patients. Due to more strict selection criteria for eligibility for transplantation, the treatment-related mortality of auto-SCT has decreased to below $5 \%$ and transplanted patients have an excellent survival after their transplant. ${ }^{5-7}$

In the previous HOVON (Haemato-Oncology Foundation for Adults in the Netherlands) 41 study we examined a two-step approach consisting of non-intensive induction therapy followed by HDM and auto-SCT. ${ }^{8}$ Although the vincristine-adriamycin-dexamethasone (VAD) induction treatment was considered too toxic, the survival of patients was good with a median survival of 8 years from registration and, for the transplanted patients, a median survival of 10 years from the date of transplantation. Other retrospective single-center studies have also examined the value of various induction regimens before HDM and auto-SCT and concluded that induction therapy is associated with a better overall survival (OS), with possibly the greatest benefit for patients with $>10 \%$ bone marrow plasma cell infiltration. ${ }^{9,10}$

Bortezomib (Velcade ${ }^{\circledast}$ ), a proteasome inhibitor frequently used in first-line and relapse treatment in patients with multiple myeloma (MM), has been given to newly diagnosed and relapsed AL amyloidosis patients in mostly retrospective and only one prospective multicenter study. The hematologic response rate to bortezomib was excellent, ranging from $50 \%$ to $80 \%$ with a CR rate from $25 \%$ to $47 \%$; furthermore, the CR occurred rapidly, especially when the drug was administered bi-weekly.11-13 Common toxicities included thrombocytopenia, peripheral sensory neuropathy, neuropathic pain, hypotension and peripheral edema.

Considering the potent effect of bortezomib in relapsed $\mathrm{AL}$ amyloidosis patients and the improvement in response rates achieved when used as first-line treatment in MM patients, we hypothesized that the use of this drug, in combination with auto-SCT, could also improve the response rates of $\mathrm{AL}$ amyloidosis patients. Because the $\mathrm{CR}$ rate, in particular, is closely related to progression-free survival (PFS), OS and organ responses, the current trial investigated the efficacy of induction treatment consisting of bortezomib and dexamethasone followed by HDM and auto-SCT to improve the CR rate after auto-SCT.

\section{Methods}

The HOVON 104 study was conducted in 15 centers in the Netherlands, Germany, and Belgium. The trial started with a randomized, phase III design but due to slow accrual the dexamethasone arm closed after including seven patients and the trial continued as a single-arm, phase II study.

Patients with biopsy-proven, systemic, AL amyloidosis, aged between 18-70 years, with detectable M-protein and/or involved free light chains (FLC) $>50 \mathrm{mg} / \mathrm{L}$, World Health Organization (WHO) Performance Status 0-2, and New York Heart Association (NYHA) stage 1-2 were included. Eligibility criteria at inclusion corresponded to eligibility criteria for auto-SCT with the exception of measurement of cardiac ejection fraction. Major exclusion criteria were concurrent MM defined as SalmonDurie stage II or III, previous treatment of plasma cell dyscrasia, symptomatic orthostatic hypotension, symptomatic effusions, a N-terminal pro-brain natriuretic peptide (NT-proBNP) level $>5,000 \mathrm{pg} / \mathrm{mL}$, troponin $\mathrm{T}>0.06$ $\mu \mathrm{g} / \mathrm{L}$ or troponin I $>2 \mathrm{x}$ upper limit of normal, estimated glomerular filtration rate $(e G F R)<30 \mathrm{~mL} / \mathrm{min} / 1.73 \mathrm{~m}^{2}$, National Cancer Institute Common Terminology Criteria for Adverse Events (NCI CTCAE) sensory peripheral neuropathy $>$ grade 2 or $>$ grade 1 with pain and motor peripheral neuropathy > grade 2 (see the Online Supplement for the complete list). Inclusion and exclusion criteria were also established for stem cell mobilization. Inclusion criteria comprised a WHO Performance Status $0-2$, NYHA stage $1-2$, and ejection fraction $>45 \%$. The exclusion criteria are listed in the Online Supplement. The study was approved by the ethics committee of all participating hospitals and the University Medical Center Utrecht (Institutional Review Board n. 10-426). All procedures were conducted in compliance with the Declaration of Helsinki. Written informed consent to participation in the study was provided by all patients (EudraCT number 2010-021445-42).

\section{Treatment design}

Four 21-day cycles of induction treatment were given. The cycles consisted of bortezomib subcutaneously (sc) $1.3 \mathrm{mg} / \mathrm{m}^{2}$ on days $1,4,8$, and 11 and dexamethasone $20 \mathrm{mg}$ orally on each day of bortezomib administration and the following day. Dose adjustments are described in the Online Supplement. Stem cell mobilization began within 4-6 weeks after the start of the last induction cycle using granulocyte colony-stimulating factor 10 $\mu \mathrm{g} / \mathrm{kg}$ divided in two doses, given for 5 days. The melphalan dosage was $200 \mathrm{mg} / \mathrm{m}^{2}$ given in 2 days. Patients with an eGFR $<40 \mathrm{~mL} / \mathrm{min} / 1.73 \mathrm{~m}^{2}$ were given $100 \mathrm{mg} / \mathrm{m}^{2}$ melphalan. Hematologic response was measured after each induction cycle and it was planned that all patients would receive HDM. Patients not responding to induction treatment could proceed directly to stem cell mobilization and auto-SCT if eligibility criteria were met.

\section{Hematologic and organ response criteria}

Organ involvement and hematologic and organ responses were evaluated according to the consensus criteria of the International Society of Amyloidosis published in 2005 with some modifications such as the addition of a VGPR category and addition of NT-proBNP for cardiac response ${ }^{14,15}$ (see the Online Supplement).

Both hematologic and organ responses were measured after 
each induction cycle, after stem cell mobilization and before autoSCT, and thereafter every 3 months for 5 years after registration. In addition, patients could participate in a side study with measurement of minimal residual disease by flow cytometry when a CR was reached or 6 months after auto-SCT. Flow cytometry was performed centrally with a sensitivity level of $10^{-5}$. Details are provided in the Online Supplement.

\section{Statistical design and endpoints}

The primary endpoint was the efficacy of bortezomib-dexamethasone induction treatment followed by HDM and auto-SCT, measured as the proportion of patients with a CR at 6 months after auto-SCT. The secondary endpoints and analysis of the effect of baseline characteristics on auto-SCT, OS and PFS are described in the Online Supplement.

\section{Results}

\section{Patients' characteristics}

Between March 2012 and April 2016, 50 patients were enrolled in the phase II part of the trial. Table 1 summa-

Table 1. Baseline characteristics of 50 patients, separated for groups that did or did not proceed to autologous stem cell transplantation.

\begin{tabular}{|c|c|c|c|}
\hline Patient & $\begin{array}{l}\text { who proceeded to auto-SCT } \\
\qquad(\mathrm{N}=35)\end{array}$ & $\begin{array}{l}\text { Patients who did not proceed to auto SCT } \\
\qquad(\mathrm{N}=15)\end{array}$ & $\begin{array}{l}\text { All patients } \\
(\mathrm{N}=50)\end{array}$ \\
\hline Age (years), median (IQR) & $59(50-63)$ & $60(53-63)$ & $59(51-63)$ \\
\hline Sex (female), n (\%) & $14(40 \%)$ & $6(40 \%)$ & $20(40 \%)$ \\
\hline $\begin{array}{l}\text { WHO performance status, } \mathrm{n}(\%) \\
0 \\
1 \\
2\end{array}$ & $\begin{array}{c}17(49 \%) \\
16(46 \%) \\
1(3 \%)\end{array}$ & $\begin{array}{l}6(40 \%) \\
5(33 \%) \\
4(27 \%)\end{array}$ & $\begin{array}{c}23(46 \%) \\
21(42 \%) \\
5(10 \%)\end{array}$ \\
\hline $\begin{array}{l}\text { Clonal disease, } n(\%) \\
\text { Lambda } \\
\text { Kappa }\end{array}$ & $\begin{array}{l}27(77 \%) \\
8(23 \%)\end{array}$ & $\begin{array}{l}13(87 \%) \\
2(13 \%)\end{array}$ & $\begin{array}{l}40(80 \%) \\
10(20 \%)\end{array}$ \\
\hline Involved FLC level, median (IQR) & $167(63-341)$ & $205(64-493)$ & $181(73-35)$ \\
\hline dFLC, median (IQR) & $214(103-342)$ & $212(68-461)$ & $213(80-397)$ \\
\hline $\begin{array}{l}\text { Number of patients with } \mathrm{dFLC} \geq 180 \mathrm{mg} / \mathrm{L} \text {, } \\
\text { median (IQR) }\end{array}$ & $17(49 \%)$ & $8(53 \%)$ & $25(50 \%)$ \\
\hline$\%$ plasma cells in bone marrow, median (IQR) & $6(4-11)$ & $5(2-8)$ & $6(0-33)$ \\
\hline $\begin{array}{l}\text { Number of patients with } \geq 10 \% \text { plasma cells, } \\
\text { median (IQR) }\end{array}$ & $12(34 \%)$ & $2(13 \%)$ & $14(28 \%)$ \\
\hline Median number of organs involved, $\mathrm{n}(\%)$ & $2(1-3)$ & $2(1-3)$ & $2(1-3)$ \\
\hline $\begin{array}{l}\text { Number of organs involved } \\
\geq 2 \\
\geq 3\end{array}$ & $\begin{array}{l}26(74 \%) \\
12(34 \%)\end{array}$ & $\begin{array}{l}10(67 \%) \\
7(47 \%)\end{array}$ & $\begin{array}{l}36(72 \%) \\
19(38 \%)\end{array}$ \\
\hline $\begin{array}{l}\text { Organ involvement, n (\%) } \\
\text { Kidney } \\
\text { Heart } \\
\text { Nervous system } \\
\text { Gastrointestinal system } \\
\text { Liver } \\
\text { Soft tissues }\end{array}$ & $\begin{array}{c}29(83 \%) \\
23(66 \%) \\
4(11 \%) \\
4(11 \%) \\
8(23 \%) \\
5(14 \%)\end{array}$ & $\begin{array}{c}12(80 \%) \\
10(67 \%) \\
3(20 \%) \\
0(0 \%) \\
5(33 \%) \\
3(20 \%)\end{array}$ & $\begin{array}{c}41(82 \%) \\
33(66 \%) \\
7(14 \%) \\
4(8 \%) \\
13(26 \%) \\
8(16 \%)\end{array}$ \\
\hline $\begin{array}{l}\text { Mayo classification, n (\%) } \\
\text { I } \\
\text { II } \\
\text { III }\end{array}$ & $\begin{array}{l}12(34 \%) \\
10(29 \%) \\
12(34 \%)\end{array}$ & $\begin{array}{l}4(27 \%) \\
6(40 \%) \\
5(33 \%)\end{array}$ & $\begin{array}{l}16(32 \%) \\
16(32 \%) \\
17(34 \%)\end{array}$ \\
\hline $\begin{array}{l}\text { NYHA stage, n (\%) } \\
\text { I } \\
\text { II }\end{array}$ & $\begin{array}{l}22(63 \%) \\
12(34 \%)\end{array}$ & $\begin{array}{l}6(40 \%) \\
9(60 \%)\end{array}$ & $\begin{array}{l}28(56 \%) \\
21(42 \%)\end{array}$ \\
\hline NT-proBNP level (ng/L), median (IQR) & $675(166-1638)$ & $1110(264-2292)$ & $832(204-1638)$ \\
\hline $\begin{array}{l}\text { Echo cardiography, median (IQR) } \\
\text { Mean left ventricular wall thickness } \\
\text { Ejection fraction } \\
\text { Diastolic dysfunction }\end{array}$ & $\begin{array}{l}12 \mathrm{~mm}(11-15) \\
63 \%(55-71) \\
60 \% \text { present }\end{array}$ & $\begin{array}{l}13 \mathrm{~mm}(12-14) \\
58 \%(53-66) \\
60 \% \text { present }\end{array}$ & $\begin{array}{l}13 \mathrm{~mm}(11-15) \\
60 \%(55-68) \\
60 \% \text { present }\end{array}$ \\
\hline eGFR (mL/min/l.73 m²), median (IQR) & $68(58-87)$ & $90(60-95)$ & $72(59-90)$ \\
\hline $\begin{array}{l}\text { Renal stage } \\
\text { I } \\
\text { II } \\
\text { III }\end{array}$ & $\begin{array}{l}12(34 \%) \\
19(54 \%) \\
4(11 \%)\end{array}$ & $\begin{array}{l}8(53 \%) \\
6(40 \%) \\
1(7 \%)\end{array}$ & $\begin{array}{c}20(40 \%) \\
25(50 \%) \\
5(10 \%)\end{array}$ \\
\hline
\end{tabular}

Auto-SCT: autologous stem cell transplantation; WHO: World Health Organization; FLC: free light chain; dFLC: difference between involved and uninvolved free light chains; NYHA: New York Heart Association; NT-proBNP: N-terminal pro-brain natriuretic peptide; eGFR: estimated glomerular filtration rate. 
rized the patients' characteristics separately for those who proceeded to auto-SCT and those who did not. The median age of all the patients included was 59 years [interquartile range (IOR), 51-63]. The median number of organs involved was two (range, 1 to 5). Two or more organs were involved in 36 patients $(72 \%)$ and three or more in $19(18 \%)$. The kidney was the organ most frequently involved, with renal disease in $82 \%$ of patients, followed by the heart (in $66 \%$ of patients) and liver (in $26 \%$ of patients). The median eGFR at inclusion was 72 $\mathrm{mL} / \mathrm{min} / 1.73 \mathrm{~m}^{2}$ (IOR, 59-90) and 20, 25 and 5 patients had renal stage I, II or III, respectively. ${ }^{16}$ Mayo cardiac stage was I, II and III for 16, 16 and 17 patients, respectively (data unavailable for 1 patient). ${ }^{17}$ The initial median NT-proBNP level was 832 pg/mL (IOR, 204-1638). Echocardiography data were available for 47 patients.

Fourteen patients $(28 \%)$ had a plasma cell infiltration of $\geq 10 \%$ in the bone marrow. Most patients presented with $\lambda$ light chains (80\%). The median concentration of involved FLC was $180 \mathrm{mg} / \mathrm{L}$ (IOR, 73-352) for $\lambda$ light chains and $169 \mathrm{mg} / \mathrm{L}$ (IOR, 61-879) for $\kappa$ light chains. The median difference between involved and uninvolved FLC (dFLC) was $213 \mathrm{mg} / \mathrm{L}$ (IOR, 80-397) and 50\% of patients had a dFLC $\geq 180 \mathrm{mg} / \mathrm{L}$. Of note, seven patients were included without an M-protein or dFLC value that could qualify them for the PR or VGPR category and three of these patients also did not have a urine protein electrophoresis $>0.1 \mathrm{~g} /$ day.

In the group of seven patients enrolled in the dexamethasone arm of the closed, phase III part of the trial one patient was not eligible. The median age of the other six patients was 57 years and these patients' other baseline characteristics were also comparable to those of the 50 patients in the phase II part (data not shown). Three patients underwent auto-SCT and the estimated 3-year PFS was $83 \%$. These six patients are not included in this final analysis.

\section{Treatment characteristics}

All patients started with induction treatment. Most patients $(88 \%)$ received four cycles of bortezomib-dexamethasone, two patients received three cycles, two patients received only two cycles and two patients had to discontinue induction treatment after the first cycle. Half of the patients had dose modifications in the administered cycles as prescribed per protocol: in $50 \%$ of patients the bortezomib dose was modified and in $44 \%$ the dexamethasone dose was modified. The reasons for dose modifications for bortezomib were mostly neurotoxicity, both sensory and autonomic neuropathy (9 patients) and infection (3 patients) and for dexamethasone heart failure ( 4 patients), edema ( 3 patients), infection ( 3 patients) and myopathy (2 patients).

Eleven patients did not have their stem cells collected after induction therapy. Five patients did not fulfill the eligibility criteria, including two patients who stopped induction due to progressive heart failure and kidney failure. Two patients died, one during the first cycle of induction, three patients experienced bortezomib-related toxicity and were taken off the trial during induction treatment (1 patient with bronchial hyperreactivity, 1 patient with gastrointestinal necrosis and 1 patient with $\mathrm{NCI}$ CTCAE grade 4 sensory neuropathy) and one patient refused stem cell collection.

The median number of stem cells collected was $6.3 \times 10^{6}$
CD34/kg (IOR, 4.6-9.3). Ten patients needed 2 days of apheresis and cyclophosphamide was given to nine patients according to local policy. After stem cell collection, four patients did not proceed to HDM and stem cell reinfusion. All four had clinical deterioration: two patients developed symptomatic pleural effusions, one patient started dialysis and one patient had several toxicities, a worsening clinical condition and was taken off the trial by decision of the treating physician.

In total 35 patients (70\%) received HDM and stem cell reinfusion as an inpatient procedure. Thirty-one patients were given a full dose of HDM, per protocol; in four patients the melphalan dose was reduced to $100 \mathrm{mg} / \mathrm{m}^{2}$ because their eGFR was $<40 \mathrm{~mL} / \mathrm{min} / 1.73 \mathrm{~m}^{2}$. All patients engrafted without granulocyte colony-stimulating factor support in a median time of 13 days for white blood cells and 16 days for platelets. A CONSORT diagram summarizes the treatment course of all 50 patients (Figure 1).

Based on a univariate logistic regression model we could not identify a prognostic baseline characteristic such as NYHA stage, NT-proBNP, Mayo stage, $\geq 10 \%$ plasma cells, dFLC $\geq 180 \mathrm{mg} / \mathrm{L}$, or number of involved organs that was related to proceeding to auto-SCT after induction therapy. As shown in Table 1 the baseline Mayo stage was not statistically different between the groups of patients who did or did not proceed to autoSCT $(P=0.80)$. In the 35 patients who underwent autoSCT, 22 received full-dose bortezomib and 23 full-dose dexamethasone. In contrast, in the 15 patients who did not proceed to auto-SCT only three received full-dose bortezomib and five full-dose dexamethasone. Because troponin levels were only measured at baseline, a Mayo stage assessment could not be calculated after induction treatment or before auto-SCT.

\section{Hematologic responses}

Hematologic responses are summarized in Table 2. The overall response rate (ORR) after induction treatment was $80 \%$, which included the $38 \%$ of patients with a VGPR and $20 \%$ with a CR. The median time to first response was 28 days (IOR, 21-43) and the median time to best response was 67 days (IOR, 28-240). Eighty percent of the responses were detected within the first 3 months after starting treatment (Figure 2$)$. Ten patients $(20 \%)$ had a CR after induction treatment and eight of them proceeded to auto-SCT. Responses assessed 6 months after auto-SCT, which was the primary endpoint of the study, improved but were assessed in 35 patients only, since 15 patients did not proceed to auto-SCT. In these 35 patients, the CR rate doubled from $23 \%$ after induction treatment to $46 \%$ at 6 months after auto-SCT. The ORR increased to $86 \%$ at 6 months after auto-SCT. In the intention-to-treat analysis the CR rate at 6 months after auto-SCT was 32\% and therefore the primary endpoint of the study was not met. In the group of 15 patients who did not proceed to auto-SCT, ten $(67 \%)$ had a hematologic response. In univariate logistic regression analyses a dFLC $\geq 180 \mathrm{mg} / \mathrm{L}$ and bone marrow plasma cell infiltration $\geq 10 \%$ at diagnosis were not related to the depth of the hematologic response.

\section{Flow cytometry}

Samples for flow cytometry were available at diagnosis for 26 patients. Of this group, 20 patients proceeded to auto-SCT. The median percentage of clonal plasma cells 


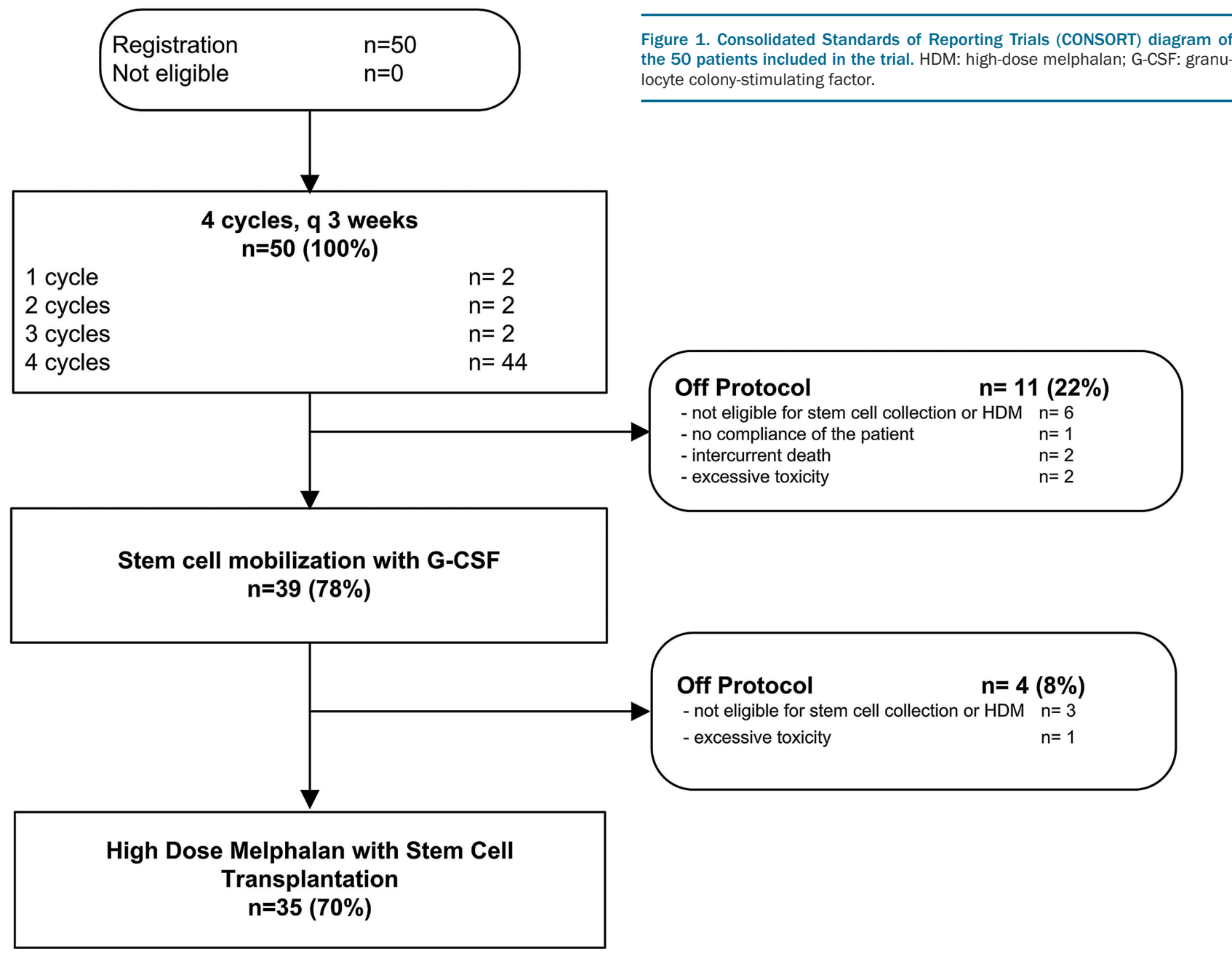

Table 2. Hematologic response rates during the different treatment phases.

\begin{tabular}{|c|c|c|c|c|}
\hline & $r$ induction therapy & $\begin{array}{c}+6 \text { months after auto-SCT } \\
(n=35)\end{array}$ & $\begin{array}{l}+12 \text { months after auto-SCT } \\
(n=34)\end{array}$ & $\begin{array}{l}+24 \text { months after auto-SCT } \\
(n=33)\end{array}$ \\
\hline \multicolumn{5}{|c|}{ Hematologic response (intention to treat) } \\
\hline $\begin{array}{l}\geq \mathrm{PR}(\mathrm{ORR}) \\
\geq \mathrm{VGPR} \\
\mathrm{CR}\end{array}$ & $\begin{array}{l}83 \%(80 \%) \\
66 \%(58 \%) \\
23 \%(20 \%)\end{array}$ & $\begin{array}{l}86 \%(60 \%) \\
72 \%(50 \%) \\
46 \%(32 \%)\end{array}$ & $\begin{array}{l}89 \%(60 \%) \\
77 \%(52 \%) \\
56 \%(38 \%)\end{array}$ & $\begin{array}{l}91 \%(60 \%) \\
76 \%(50 \%) \\
58 \%(38 \%)\end{array}$ \\
\hline Median time to first response & 28 days & & & \\
\hline Median time to best response & 67 days & & & \\
\hline
\end{tabular}

detected at baseline was $1.4 \%$ (range, 0.17-4.9). At 6 months after auto-SCT, samples were collected from seven patients. Six of these patients had a CR and were also negative for minimal residual disease. Additional analyses were performed, such as those previously reported by Perez-Persona et al. who described that a ratio of clonal plasma cells within the total bone marrow plasma cells (aPC/BMPC) $\geq 95 \%$ had prognostic value for progression to $\mathrm{MM}$ in patients with monoclonal gammopathy of unknown significance and smoldering MM. ${ }^{18}$ In the current study patients with $\mathrm{aPC} / \mathrm{BMPC} \geq 95 \%$ at baseline had a shorter PFS (hazard ratio $8.44,95 \%$ confidence interval: 1.05-67.96) and a lower probability of proceeding to auto-SCT; however, given the small sample size this was not statistically significant (odds ratio 0.16 , 95\% confidence interval: 0.02-1.67).

\section{Organ responses}

Organ responses were already seen after induction treatment and improved after HDM plus auto-SCT and are summarized in Figure 3 for both patients who proceeded to auto-SCT and those who did not. The rate of 


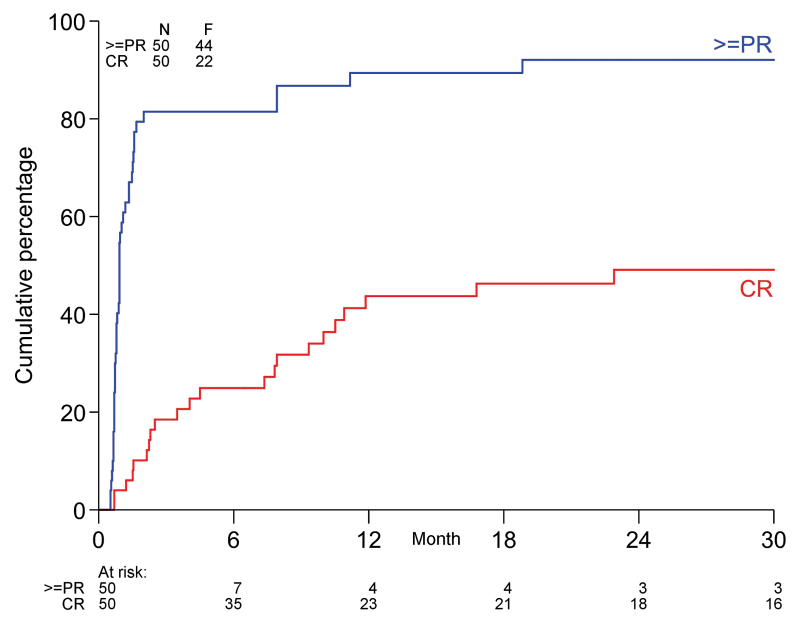

Figure 2. Response rates following the initiation of treatment. The upper line represents the time to first response, defined as partial response or better, while the lower line represents the time to first complete response. PR: partial response; CR: complete response.

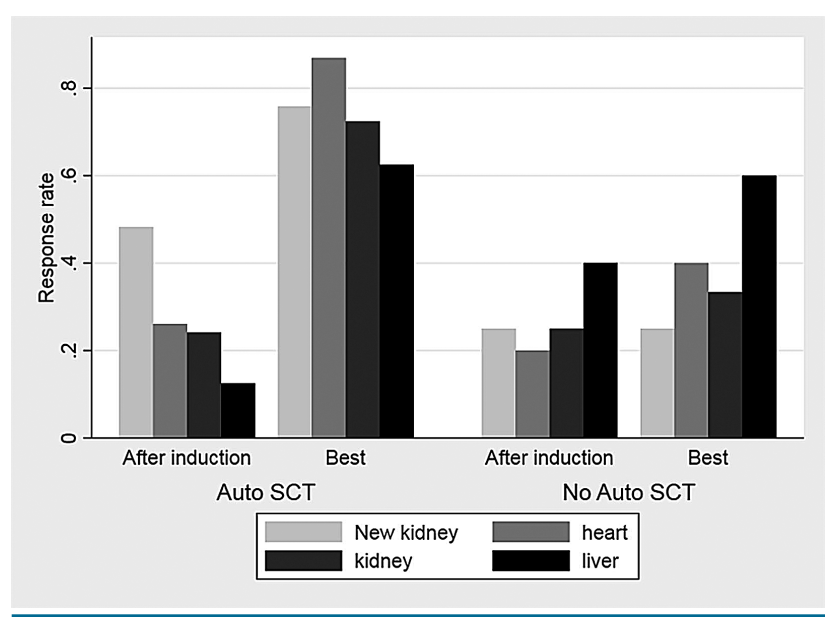

Figure 3. Organ responses depicted separately for patients who proceeded to autologous stem cell transplantation or not. Organ responses were assessed according to consensus criteria after induction and as best responses achieved during study treatment and follow-up. Auto-SCT: autologous stem cell transplantation.

(a)

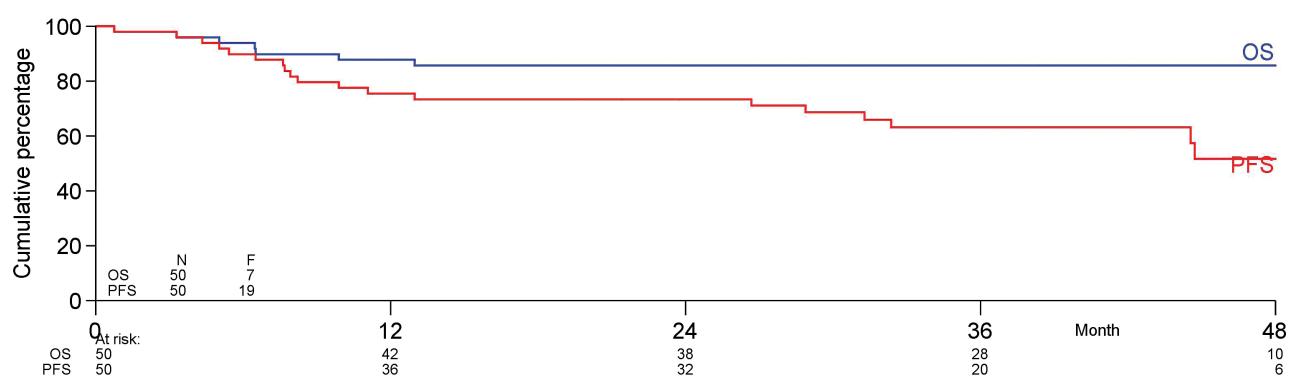

(b)

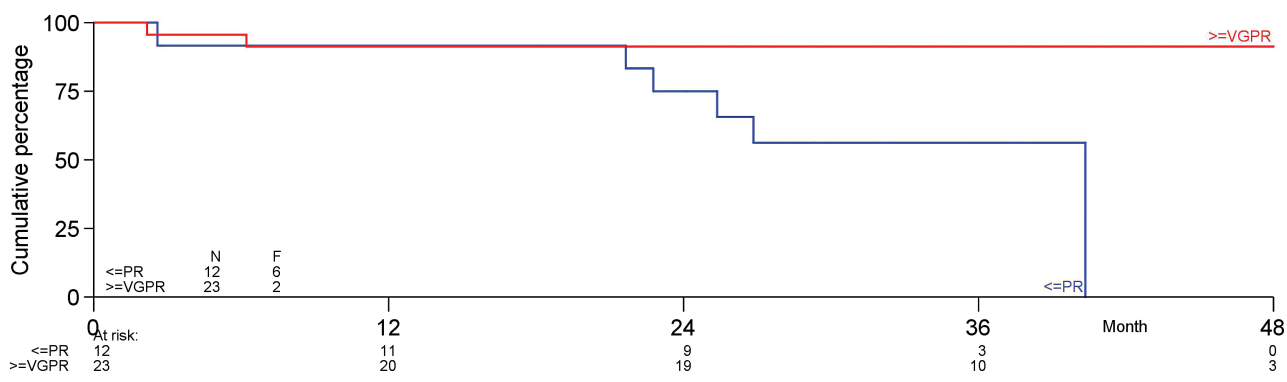

Figure 4. Overall and progression-free survival of the total cohort of patients and the 35 patients who underwent autologous stem cell transplantation. (A) Progression-free and overall survival from date of registration in the trial for the 50 patients included. (B) Progression-free survival from autologous stem cell transplantation of the 35 transplanted patients according to response achieved after induction therapy. PFS: progression-free survival; OS: overall survival; PR: partial remission; VGPR: very good partial remission.

kidney responses improved from $24 \%$ to $69 \%$ at 2 years after auto-SCT and that of cardiac responses from $24 \%$ to $78 \%$. In intention-to-treat analysis $61 \%$ of patients achieved a renal response, $72 \%$ a cardiac response and $62 \%$ a liver response. During the HDM and auto-SCT procedure two patients had a deterioration of their renal function, defined as a decrease of $25 \%$ in eGFR, which persisted during the follow-up. The median time to first organ response was 222 days for the heart (IOR, 125-395) and 318 days (IOR 91-615) for the kidney. Organ progression was seen in six of 33 patients with cardiac involve- ment, 13 out of 41 patients with kidney involvement, and three of 13 patients with liver involvement.

During the study new renal response criteria were developed $(\geq 30 \%$ decrease in proteinuria or drop of proteinuria $<0.5 \mathrm{~g} /$ day in the absence of renal progression, defined as a $\geq 25 \%$ decrease in eGFR). According to these criteria 14 patients (48\%) who proceeded to auto-SCT had a renal response after induction treatment and this increased to 22 patients (76\%) after auto-SCT (Figure 3).

Patients with a deep hematologic response (CR/VGPR) at 6 months after auto-SCT and at subsequent time- 
points had a higher renal response rate $(72 \%$ vs. $28 \%$, $P=0.03)$. Although more patients in this group also had a higher cardiac response rate (65\% vs. $35 \%)$, the difference was not statistically significant for cardiac response at any time-point after auto-SCT.

\section{Overall survival and progression-free survival}

The median follow-up of the 43 patients still alive is 38.3 months (IOR, 34-46) while that of all 50 patients is 36.9 months (IOR, 29-46). Five patients died during the follow-up, in most cases due to amyloid-related organ failure. None of the transplanted patients has died. Kaplan-Meier PFS curves for all 50 patients and the 35 transplanted patients are shown in Figure 4. The median $\mathrm{OS}$ and PFS were not reached. The 3-year estimated OS and PFS rates are $86 \%$ and $63 \%$, respectively. In total seven patients had progression of their plasma cell dyscrasia after auto-SCT, including one patient with a previous CR, three with a VGPR, two with a PR and one patient who did not have any response. None of the eight patients with a CR before auto-SCT has progressed, whereas eight of the patients who had not achieved a CR prior to auto-SCT have done so $(P=0.13)$. Using univariate Cox regression prognostic baseline characteristics, such as type of hospital (high $v s$. low number of included patients), eGFR ( $>30$ and $<50$ vs. $\geq 50 \mathrm{~mL} / \mathrm{min} / 1.73 \mathrm{~m}^{2}$, NYHA class (I vs. II), NT-proBNP (as a continuous variable), plasma cell infiltration $(<10 \%$ vs. $\geq 10 \%)$, dFLC $<180 \mathrm{mg} / \mathrm{L} v s . \geq 180 \mathrm{mg} / \mathrm{L}$, number of organs involved ( $\leq 2$ vs. $>2$ ), Mayo stage, nervous system involvement and cardiac involvement were tested but none of these variables was statistically related to OS.

\section{Adverse events and mortality}

Adverse events were commonly seen during induction treatment in all patients. The most frequent adverse events seen during induction treatment are summarized in Table 3. The most commonly experienced adverse events were neurotoxicity, gastrointestinal symtpoms, infections and cardiac disorders. Although dizziness, orthostatic hypotension and syncope may also have cardiac origins, all these events were grouped together as autonomic neuropathic events if no cardiac cause, such as arrhythmias or deterioration in ejection fraction, was detected. In total, $10 \%$ of patients experienced autonomic neuropathy and $34 \%$ had sensory neuropathy related to bortezomib. Interestingly, patients with nervous system involvement at the start of therapy did not require more dose adjustments for bortezomib compared to patients without nervous system involvement. No engraftment syndrome was seen after auto-SCT.

Overall, 47 serious adverse events were reported in 29 patients, 34 during induction treatment, three during stem cell mobilization and ten following 30 days after HDM and auto-SCT. The serious adverse events were mostly due to hospitalization (81\%) and 28 were considered related to the treatment. Twenty-nine serious adverse events resolved completely, the others were ongoing. Two patients died during the study treatment phase. One patient had a sudden cardiac death during the first induction cycle, probably related to the cardiac amyloidosis, and one patient died due to hepato-renal syndrome after receiving four cycles of induction treatment. There were no cases of transplant-related mortality.

\section{Discussion}

The aim of the current prospective, multicenter study was to investigate whether a more effective induction regimen before HDM and auto-SCT could lead to better outcomes in newly diagnosed AL amyloidosis patients. Bortezomib is considered the first drug of choice both because of the high response rates that it induces and the fact that the responses tend to occur rapidly. ${ }^{19}$ We therefore hypothesized that using a two-step approach with effective induction, starting immediately after diagnosing and staging the AL amyloidosis, would not only improve response rates after auto-SCT but would also rescue more patients from amyloidosis-related organ damage.

The ORR of $80 \%$, with $20 \%$ CR, 38\% VGPR and $22 \%$ $\mathrm{PR}$ rates achieved after induction treatment, is indeed comparable to that reported previously by Reece et al. in relapsed AL amyloidosis patients (ORR 66.7\%). ${ }^{13}$ In their study a median of six cycles of bi-weekly bortezomib was given compared to the four cycles of bi-weekly bortezomib in the current study. In retrospective analyses high response rates up to $80 \%$ have been reported in both relapsed and newly diagnosed patients. ${ }^{11,12}$ We confirm a rapid time to response with median times to first response of 28 days and to best response of 67 days.

Because of these fast and deep responses we expected

Table 3. Percentages of the most common treatment-related adverse events of bortezomib-dexamethasone induction treatment.

\begin{tabular}{|c|c|c|c|c|c|c|}
\hline Adverse event & Grade 1 & Grade 2 & Grade 3 & Grade 4 & Grade 5 & Total \\
\hline Nervous system, total & 12 & 24 & 12 & 2 & - & 50 \\
\hline - sensory PNP & 12 & 14 & 6 & 2 & - & 34 \\
\hline $\begin{array}{l}\text { - autonomic (syncope, dizziness, } \\
\text { orthostatic hypotension) }\end{array}$ & - & 6 & 4 & - & - & 10 \\
\hline - motor & - & - & 2 & - & - & 4 \\
\hline - neuropathic pain & - & 4 & - & - & - & \\
\hline $\begin{array}{l}\text { Gastrointestinal (constipation, diarrhea, } \\
\text { nausea, vomiting) }\end{array}$ & na & 30 & 10 & 4 & - & 44 \\
\hline Infections & na & 20 & 10 & - & - & 30 \\
\hline Cardiac & na & 12 & 12 & 2 & 2 & 28 \\
\hline Metabolic/nutrition & na & 18 & 6 & - & - & 24 \\
\hline Fatigue & na & 10 & 2 & - & - & 12 \\
\hline
\end{tabular}

PNP: polyneuropathy, na:not assessed 
that a higher proportion of patients would be able to proceed to HDM and auto-SCT. However, the discontinuation rate of $30 \%$ in the current study was similar to the $33 \%$ observed in the previous HOVON 41 study in which VAD induction therapy was given. ${ }^{8}$ The discontinuation rate was substantially higher than those in two other prospective, single-center studies in which bortezomib-dexamethasone induction was given prior to HDM and autoSCT $(0 \%$ and $14 \%){ }^{20,21}$ These differences could be explained by different treatment designs; for example, in the studies by Sanchorawala et al. and Huang et al., only two cycles of bortezomib and dexamethasone induction were given, instead of four. Perhaps more importantly, these were single-center studies in large, experienced hospitals, while the HOVON 104 trial was performed in 16 hospitals, which enrolled between one and eight patients per site which may impair the quality of care. We therefore think that our prospective data may better represent the real-life outcome of patients following first-line AL amyloidosis treatment. Although the discontinuation rate could not be assessed in other retrospective studies because only transplanted patients were included, these studies do suggest that induction regimens can be beneficial in patients by inducing deeper hematologic responses and better OS. ${ }^{9,10}$

The reasons for 15 patients not proceeding to HDM and auto-SCT were mostly non-eligibility according to protocol, generally caused by symptomatic effusions and poor Performance Status. Some patients had bortezomib-related toxicity and in four patients the physician decided that the patient should not proceed to auto-SCT because of organ progression. The other toxicities seen during induction treatment are summarized in Table 2. These toxicities are comparable to those reported by Reece et al., and most involved the gastrointestinal tract, heart, and nervous system or were infections. ${ }^{13}$ Although $78 \%$ of patients received four cycles of bortezomib-dexamethasone, half of the patients needed dose reductions of bortezomib and $44 \%$ needed reductions of dexamethasone. Due to the multiple organ dysfunctions typically seen in AL amyloidosis, these patients do not tolerate the same chemotherapy schedules as MM patients do. This also seems to hold true for the "fittest" patients who appeared to be eligible for auto-SCT at diagnosis. Encouraging data from retrospective analyses illustrate that other bortezomib-based regimens with reduced doses of bortezomib to $1.0 \mathrm{~m} / \mathrm{m}^{2}$ bi-weekly or $1.5 \mathrm{mg} / \mathrm{m}^{2}$ once weekly, combined with dexamethasone and cyclophosphamide once weekly, could maintain the high response rates but data on toxicity are limited. ${ }^{22,23}$ Once weekly dosing of bortezomib could therefore be the preferred schedule in AL amyloidosis patients.

Using flow cytometry analysis at diagnosis we identified a negative association between aPC/BMPC $\geq 95 \%$ and PFS. In addition, we found that patients with $\mathrm{aPC} / \mathrm{BMPC} \geq 95 \%$ had a lower probability of proceeding to auto-SCT; however, due to our small sample size of 26 patients this was not statistically significant. A high ratio of clonal plasma cells may reflect a more aggressive plasma cell clone in the bone marrow and its prognostic value has been determined in patients with monoclonal gammopathy of undetermined significance and smoldering MM. ${ }^{18}$ In patients with $\mathrm{AL}$ amyloidosis it has been demonstrated that the persistence of $5 \%$ or more normal plasma cells at diagnosis was related to OS, but due to the shorter follow-up and excellent survival in the current study, this association could not be confirmed. ${ }^{24}$ The small sample size of the current study could perhaps be the reason that we could not confirm that other baseline characteristics, such as $\geq 10 \%$ plasma cells or a dFLC $\geq 180$ $\mathrm{mg} / \mathrm{L}$, were negatively associated with survival.

There was no transplant-related mortality among the 35 patients who did receive HDM and auto-SCT and the patients had an excellent outcome. This is remarkable, because a previous prospective, multicenter study performed by Jaccard et al. reported a high mortality rate of $24 \%$ related to the auto-SCT procedure. This study, like ours, was also conducted in less experienced centers. ${ }^{25}$ It could be speculated that the induction treatment before HDM and auto-SCT functions as a selection mechanism because only patients who are able to tolerate chemotherapy proceed to high-dose treatment. Indeed, more patients who received the full doses of the induction regimen without dose adjustments proceeded to auto-SCT. A randomized trial is needed to investigate the role of induction therapy before auto-SCT. Interestingly, our study demonstrates that HDM with auto-SCT is also possible for some patients with the highest cardiac Mayo risk score of III, which was present in 34\% of our study population. These patients did not experience more toxicity than the other risk groups. However since the start of this study new prognostic cardiac scoring systems have been published which may improve the selection of patients suitable for HDM and auto-SCT. ${ }^{26,27}$

The hematologic responses improved to $86 \%$ at 6 months after auto-SCT. In particular, the CR rate increased steadily after auto-SCT. Since the quality of response in AL amyloidosis patients is closely related to survival this is a very important observation. The hematologic responses after auto-SCT are less than those reported in the two other prospective, single-center trials but are better than those reported in larger retrospective cohorts. ${ }^{7,20,21,28,29}$ However, due to the high discontinuation rate before auto-SCT the primary endpoint of the study, an improvement, in intention-to-treat analysis, of the CR rate from $30 \%$ to $50 \%$ was not achieved.

Organ responses were already detected after induction therapy and the rates were $24 \%, 24 \%$ and $23 \%$ for the kidney, heart and liver, respectively. The new renal response criteria set was also prospectively evaluated and was $27 \%$ after induction treatment. ${ }^{16}$ In total, six patients developed kidney failure, defined as an eGFR $<30$ $\mathrm{mL} / \mathrm{min} / 1.73 \mathrm{~m}^{2}$ : two of them had renal stage I at diagnosis, two had stage II and two had stage III. After auto-SCT organ response rates continued to improve to 60\%-80\% (Figure 3) which are comparable to those after auto-SCT in previous reports. ${ }^{29}$

In conclusion, although the primary endpoint of the study was not met, in this first, multicenter, prospective trial with twice-weekly bortezomib, combined with dexamethasone treatment, we documented a high hematologic response rate of $80 \%$ in patients with newly diagnosed AL amyloidosis. However, these responses cannot prevent amyloidosis-related organ failure and the treatment-induced NCI-CTCAE grade 2 and higher gastrointestinal, cardiac, metabolic and neurotoxicity in more than $70 \%$ of patients, leading to a high discontinuation rate of $30 \%$ before auto-SCT. Possibly due to the unintended selection of induction treatment prior to HDM and auto-SCT, the transplants were performed without 
any mortality and improved the CR rate from $20 \%$ to $46 \%$ at 6 months after auto-SCT.

\section{Acknowledgments}

The authors would like to thank all participating patients and study centers. The authors also thank the local data managers for study coordination and collecting patients' data, in particular the study team at the HOVON Data Center, Klaartje Nijssen and Marianne Gawlik. This investigator-sponsored trial was financially supported by the Dutch Cancer Society (KWF UU2010-4884) and Janssen Cilag which provided the drug bortezomib, free of charge.

\section{References}

1. Wechalekar AD, Gillmore JD, Hawkins PN. Systemic amyloidosis. Lancet. 2016;387 (10038):2641-2654.

2. Palladini G, Dispenzieri A, Gertz MA, et al. New criteria for response to treatment in immunoglobulin light chain amyloidosis based on free light chain measurement and cardiac biomarkers: impact on survival outcomes. J Clin Oncol. 2012;30(36):45414549.

3. Gertz MA, Lacy MQ, Dispenzieri A, et al. Effect of hematologic response on outcome of patients undergoing transplantation for primary amyloidosis: importance of achieving a complete response. Haematologica. 2007;92(10):1415-1418.

4. Merlini G, Wechalekar AD, Palladini G Systemic light chain amyloidosis: an update for treating physicians. Blood. 2013;121(26): 5124-5130

5. Gertz MA, Lacy MQ, Dispenzieri A, et al. Refinement in patient selection to reduce treatment-related mortality from autologous stem cell transplantation in amyloidosis. Bone Marrow Transplant. 2013;48(4): 557-561.

6. Sanchorawala V, Sun F, Quillen K, Sloan JM, Berk JL, Seldin DC. Long-term outcome of patients with AL amyloidosis treated with high-dose melphalan and stem cell transplantation: 20-year experience. Blood. 2015;126(20):2345-2347.

7. D'Souza A, Dispenzieri A, Wirk B, et al. Improved outcomes after autologous hematopoietic cell transplantation for light chain amyloidosis: a center for international blood and marrow transplant research study. J Clin Oncol. 2015;33(32): 3741-3749.

8. Hazenberg BP, Croockewit A, van der Holt $B$, et al. Extended follow up of high-dose melphalan and autologous stem cell transplantation after vincristine, doxorubicin, dexamethasone induction in amyloid light chain amyloidosis of the prospective phase II HOVON-41 study by the Dutch-Belgian Co-operative Trial Group for Hematology Oncology. Haematologica. 2015;100(5):677682

9. Hwa YL, Kumar SK, Gertz MA, et al. Induction therapy pre-autologous stem cell transplantation in immunoglobulin light chain amyloidosis: a retrospective evaluation. Am J Hematol. 2016;91(10):984-988.

10. Afrough A, Saliba RM, Hamdi A, et al.
Impact of induction therapy on the outcome of immunoglobulin light chain amyloidosis after autologous hematopoietic stem cell transplantation. Biol Blood Marrow Transplant. 2018;24(11): 2197-2203.

11. Wechalekar AD, Lachmann HI, Offer M, Hawkins PN, Gillmore JD. Efficacy of bortezomib in systemic AL amyloidosis with relapsed/refractory clonal disease. Haematologica. 2008;93(2):295-298.

12. Kastritis E, Wechalekar AD, Dimopoulos $M A$, et al. Bortezomib with or without dexamethasone in primary systemic (light chain) amyloidosis. J Clin Oncol. 2010;28(6): 1031-1037.

13. Reece DE, Hegenbart U, Sanchorawala V, et al. Efficacy and safety of once-weekly and twice-weekly bortezomib in patients with relapsed systemic AL amyloidosis: results of a phase 1/2 study. Blood. 2011;118(4):865 873.

14. Gertz MA, Comenzo R, Falk RH, et al. Definition of organ involvement and treatment response in immunoglobulin light chain amyloidosis (AL): a consensus opinion from the 10th International Symposium on Amyloid and Amyloidosis, Tours, France, 18-22 April 2004. Am J Hematol. 2005;79 (4):319-328.

15. Comenzo RL, Reece D, Palladini G, et al Consensus guidelines for the conduct and reporting of clinical trials in systemic lightchain amyloidosis. Leukemia. 2012;26(11): 2317-2325.

16. Palladini G, Hegenbart U, Milani P, et al. A staging system for renal outcome and early markers of renal response to chemotherapy in AL amyloidosis. Blood. 2014;124(15): 2325-2332.

17. Dispenzieri A, Gertz MA, Kyle RA, et al. Prognostication of survival using cardiac troponins and $\mathrm{N}$-terminal pro-brain natriuretic peptide in patients with primary systemic amyloidosis undergoing peripheral blood stem cell transplantation. Blood. 2004;104(6):1881-1887.

18. Perez-Persona E, Vidriales MB, Mateo G, et al. New criteria to identify risk of progression in monoclonal gammopathy of uncertain significance and smoldering multiple myeloma based on multiparameter flow cytometry analysis of bone marrow plasma cells. Blood. 2007;110(7):2586-2592

19. Palladini G, Merlini G. What is new in diagnosis and management of light chain amyloidosis? Blood. 2016;128(2):159-168.
20. Huang $X$, Wang $Q$, Chen W, et al. Induction therapy with bortezomib and dexamethasone followed by autologous stem cell transplantation versus autologous stem cell transplantation alone in the treatment of renal AL amyloidosis: a randomized controlled trial. BMC Med. 2014;12:2

21. Sanchorawala V, Brauneis D, Shelton AC, et al. Induction therapy with bortezomib followed by bortezomib-high dose melphalan and stem cell transplantation for light chain amyloidosis: tesults of a prospective clinical trial. Biol Blood Marrow Transplant. 2015;21(8):1445-1451.

22. Mikhael JR, Schuster SR, Jimenez-Zepeda $\mathrm{VH}$, et al. Cyclophosphamide-bortezomibdexamethasone (CyBorD) produces rapid and complete hematologic response in patients with AL amyloidosis. Blood. 2012;119(19):4391-4394

23. Venner CP, Lane $T$, Foard D, et al. Cyclophosphamide, bortezomib, and dexamethasone therapy in AL amyloidosis is associated with high clonal response rates and prolonged progression-free survival. Blood. 2012;119(19):4387-4390.

24. Paiva B, Vidriales MB, Perez JJ, et al. The clinical utility and prognostic value of multiparameter flow cytometry immunophenotyping in light-chain amyloidosis. Blood. 2011;117(13):3613-3616.

25. Jaccard A, Moreau P, Leblond V, et al. Highdose melphalan versus melphalan plus dexamethasone for AL amyloidosis. N Engl J Med. 2007;357(11):1083-1093

26. Kumar S, Dispenzieri A, Lacy $\mathrm{MO}$, et al. Revised prognostic staging system for light chain amyloidosis incorporating cardiac biomarkers and serum free light chain measurements. J Clin Oncol. 2012;30(9):989-995.

27. Wechalekar AD, Schonland SO, Kastritis E, et al. A European collaborative study of treatment outcomes in 346 patients with cardiac stage III AL amyloidosis. Blood. 2013;121(17):3420-3427

28. Madan S, Kumar SK, Dispenzieri A, et al. High-dose melphalan and peripheral blood stem cell transplantation for light-chain amyloidosis with cardiac involvement. Blood. 2012;119(5):1117-1122

29. Cibeira MT, Sanchorawala V, Seldin DC, et al. Outcome of AL amyloidosis after highdose melphalan and autologous stem cell transplantation: long-term results in a series of 421 patients. Blood. 2011;118(16):43464352 . 